Network Working Group

Request for Comments: 5210

Category: Experimental
J. Wu

J. Bi

X. Li

G. Ren

K. Xu

Tsinghua University

M. Williams

Juniper Networks

June 2008

\title{
A Source Address Validation Architecture (SAVA) Testbed and Deployment Experience
}

Status of This Memo

This memo defines an Experimental Protocol for the Internet community. It does not specify an Internet standard of any kind. Discussion and suggestions for improvement are requested. Distribution of this memo is unlimited.

Abstract

\begin{abstract}
Because the Internet forwards packets according to the IP destination address, packet forwarding typically takes place without inspection of the source address and malicious attacks have been launched using spoofed source addresses. In an effort to enhance the Internet with IP source address validation, a prototype implementation of the IP Source Address Validation Architecture (SAVA) was created and an evaluation was conducted on an IPv6 network. This document reports on the prototype implementation and the test results, as well as the lessons and insights gained from experimentation.
\end{abstract}


Table of Contents

1. Introduction . . . . . . . . . . . . . . . . . . . . . . . 3

2. A Prototype SAVA Implementation . . . . . . . . . . . . . . . 4

2.1. Solution Overview . . . . . • . . . . . . . . . . . . . 4

2.2. IP Source Address Validation in the Access Network . . . 6

2.3. IP Source Address Validation at Intra-AS/Ingress Point . 9

2.4. IP Source Address Validation in the Inter-AS Case

(Neighboring AS) . . . . . . . . . . . . . . . . . . 9

2.5. IP Source Address Validation in the Inter-AS Case (Non-Neighboring AS) . . . . . . . . . . . . . . 12

3. SAVA Testbed . . . . . . . . . . . . . . . . . . . . . 15

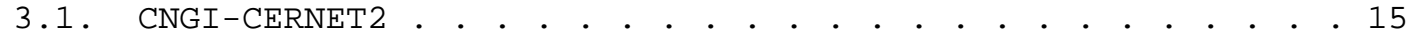

3.2. SAVA Testbed on CNGI-CERNET2 Infrastructure . . • . . . 16

4. Test Experience and Results . . . . . . . . . . . . . . . 17

4.1. Test Scenarios . . . . . . . . . . . . . . . . . 17

4.2. Test Results . . . . . . . • • • . . . . . . . . . . 18

5. Limitations and Issues . . . . . . . . . . . . . . . . 18

5.1. General Issues . . . . . . . . . . . . . . . . . . 18

5.2. Security Issues . . . . . . . . . . . . . . . . . 20

5.3. Protocol Details . . . . . . . . . . . . . . . . 20

6. Conclusion . . . . . . . . . . . . . . . . . . . . . . . 21

7. Security Considerations . . . . . . . . . . . . . . . 22

8. Acknowledgements . . . . . . . . . . . . . . . . . 22

9. References . . . . . . . . . . . . . . . . . . . . . . 23

9.1. Normative References . . . . . . . . . . . . . . . . . 23

9.2. Informative References . . . . . . . . . . . . . . 23 


\section{Introduction}

By design, the Internet forwards data packets solely based on the destination IP address. The source IP address is not checked during the forwarding process in most cases. This makes it easy for malicious hosts to spoof the source address of the IP packet. We believe that it would be useful to enforce the validity of the source IP address for all the packets being forwarded.

Enforcing the source IP address validity would help us achieve the following goals:

- Since packets which carry spoofed source addresses would not be forwarded, it would be impossible to launch network attacks that are enabled by using spoofed source addresses and more difficult to successfully carry out attacks enhanced or strengthened by the use of spoofed source addresses.

- Being able to assume that all packet source addresses are correct would allow traceback to be accomplished accurately and with confidence. This would benefit network diagnosis, management, accounting, and applications.

As part of the effort in developing a Source Address Validation Architecture (SAVA), we implemented a SAVA prototype and deployed the prototype in 12 ASes in an operational network as part of China Next Generation Internet (CNGI) Project [Wu07]. We conducted evaluation experiments. In this document, we first describe the prototype solutions and then report experimental results. We hope that this document can provide useful insights to those interested in the subject, and can serve as an initial input to future IETF effort in this area.

In recent years, there have been a number of research and engineering efforts to design IP source address validation mechanisms, such as [RFC2827], [Park01], [Li02], [Brem05], and [Snoe01]. Our SAVA prototype implementation was inspired by some of the schemes from the proposed or existing solutions.

The prototype implementation and experimental results presented in this report serve only as an input, and by no means preempt any solution development that may be carried out by future IETF effort. Indeed, the presented solutions are experimental approaches and have a number of limitations as discussed in sections 5 and 6 . 


\section{A Prototype SAVA Implementation}

\subsection{Solution Overview}

A multiple-fence solution is proposed in this document. That is, there are multiple points in the network at which the validity of a packet's source address can be checked. This is because in the current single-fence model where source address validity is essentially checked only at ingress to the network, deployment has been inadequate to the point that there is always sufficient opportunity to mount attacks based on spoofed source addresses, and it seems likely that this condition will continue in the foreseeable future. A multiple-fence solution will allow "holes" in deployment to be covered and validity of the source address to be evaluated with increased confidence across the whole Internet. The assumption here is that when validity checking is not universal, it is still worthwhile to increase the confidence in the validity of source addresses and to reduce the opportunities to mount a source address spoofing attack.

Furthermore, the architecture allows for multiple independent and loosely-coupled checking mechanisms. The motivation for this is that in the Internet at large, it is unrealistic to expect any single IP source address validation mechanism to be universally supported. Different operators and vendors may choose to deploy/develop different mechanisms to achieve the same end, and there need to be different mechanisms to solve the problem at different places in the network. Furthermore, implementation bugs or configuration errors could potentially render an implementation ineffective. Therefore, our prototype SAVA implementation is a combination of multiple coexisting and cooperating mechanisms. More specifically, we implement source IP address validation at three levels: access network source address validation; intra-As source address validation; and inter-AS source address validation, as shown in Figure 1. The system details can be found in [Wu07]. 


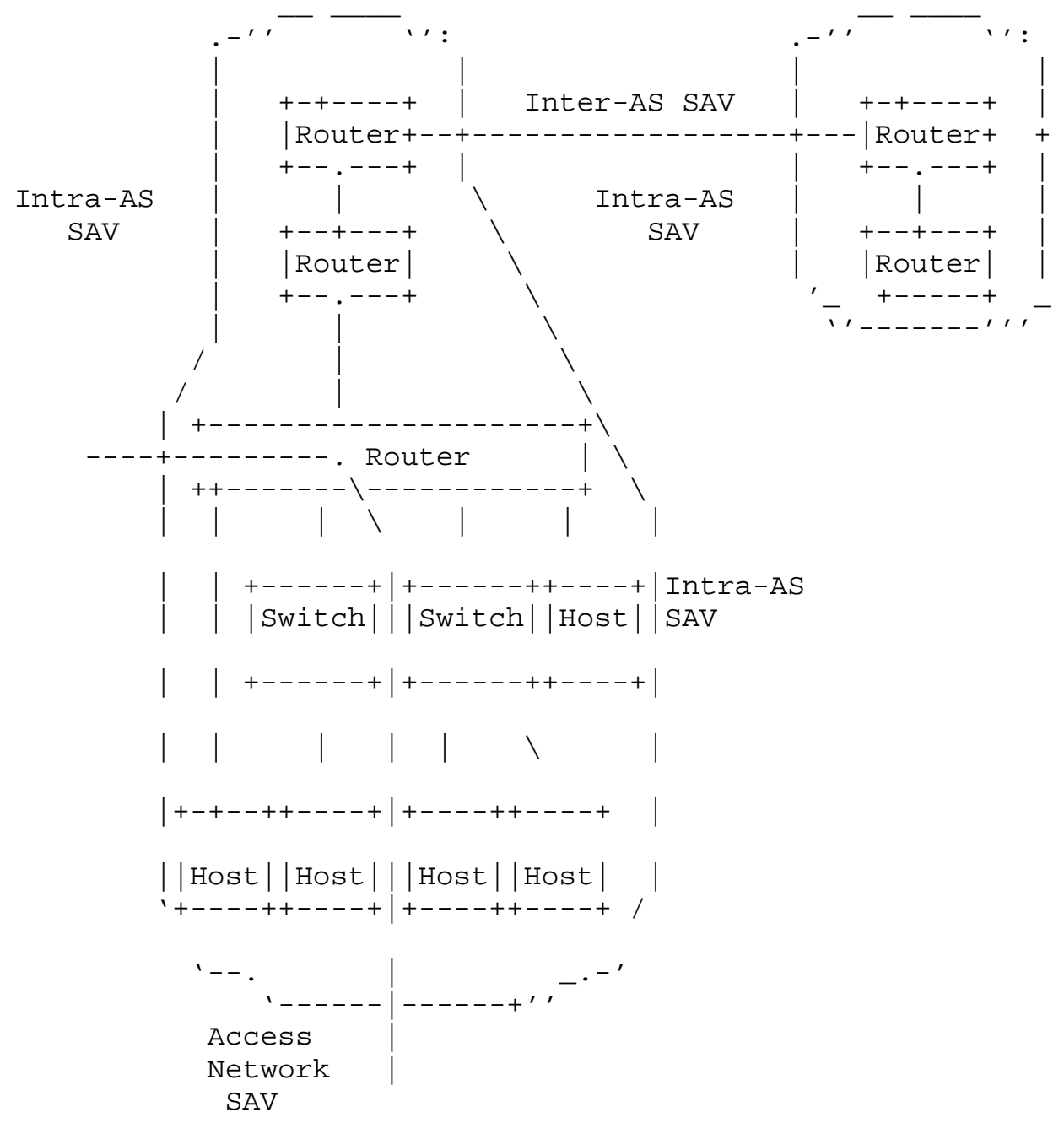

Key: SAV - Source Address Validation

Figure 1: Solution Overview

This document divides source address validation into three different classes of solutions:

1. Access network. This prevents a host in a network from spoofing the address of another host in the same network segment. This enables host-granularity of protection compared to Intra-AS prevention. See Section 2.2 for details. 
2. Intra-AS. When the edge router of an access network performs source address validation (e.g., using [RFC2827] and [RFC3704]), hosts are prevented from spoofing an arbitrary address, but unless access network SAV is employed, they may be able to spoof an address of a host in the same network segment. In a degenerate case, when a router connects a single host, the host can't spoof any address.

3. Inter-AS. Mechanisms that enforce packet source address correctness at AS boundaries. Because the global Internet has a mesh topology, and because different networks belong to different administrative authorities, IP source address validation at the Inter-AS level is more challenging. Nevertheless, we believe this third level of protection is necessary to detect packets with spoofed source addresses, when the first two levels of source address validation are missing or ineffective.

In the following sections, we describe the specific mechanisms implemented at each of the three levels in detail.

\subsection{IP Source Address Validation in the Access Network}

At the access network level, the solution ensures the host inside the access network cannot use the source address of another host. The host address should be a valid address assigned to the host statically or dynamically. The solution implemented in the experiment provides such a function for Ethernet networks. A layer-3 source address validation architecture device (SAVA Device) for the access network (the device can be a function inside the Customer Premises Equipment (CPE) router or a separate device) is deployed at the exit of the access network. Source address validation architecture agents (SAVA Agents) are deployed inside the access network. (In fact, these agents could be a function inside the first hop router/switch connected to the hosts.) A set of protocols was designed for communication between the host, SAVA Agent, and SAVA Device. Only a packet originating from the host that was assigned that particular source address may pass through the SAVA Agent and SAVA Device.

Two possible deployment variants exist; we will call them Variant A and Variant B. In Variant A, an agent is mandatory and each host is attached to the agent on a dedicated physical port. In Variant B, hosts are required to perform network access authentication and generate key material needed to protect each packet. In this variant, the agent is optional. 
The key function of Variant $A$ is to create a dynamic binding between a switch port and valid source IP address, or a binding between Media Access Control (MAC) address, source IP address, and switch port. In the prototype, this is established by having hosts employ a new address configuration protocol that the switch is capable of tracking.

Note: In a production environment, the approach in the prototype would not be sufficient due to reasons discussed in section 5 .

In Variant A, there are three main participants: Source Address Request Client (SARC) on the host, Source Address Validation Proxy (SAVP) on the switch, and Source Address Management Server (SAMS). as shown in Figure 2. The solution follows the basic steps below:

1. The SARC on the end host sends an IP address request. The SAVP on the switch relays this request to the SAMS and records the MAC address and incoming port. If the address has already been predetermined by the end host, the end host still needs to put that address in the request message for verification by SAMS.

2. After the SAMS receives the IP address request, it then allocates a source address for that SARC based on the address allocation and management policy of the access network, it stores the allocation of the IP address in the SAMS history database for traceback, then sends response message containing the allocated address to the SARC.

3. After the SAVP on the access switch receives the response, it binds the IP address and the former stored MAC address of the request message with the switch port on the binding table. Then, it forwards the issued address to SARC on the end host.

4. The access switch begins to filter packets sent from the end host. Packets which do not conform to the tuple (IP address, Switch Port) are discarded. 


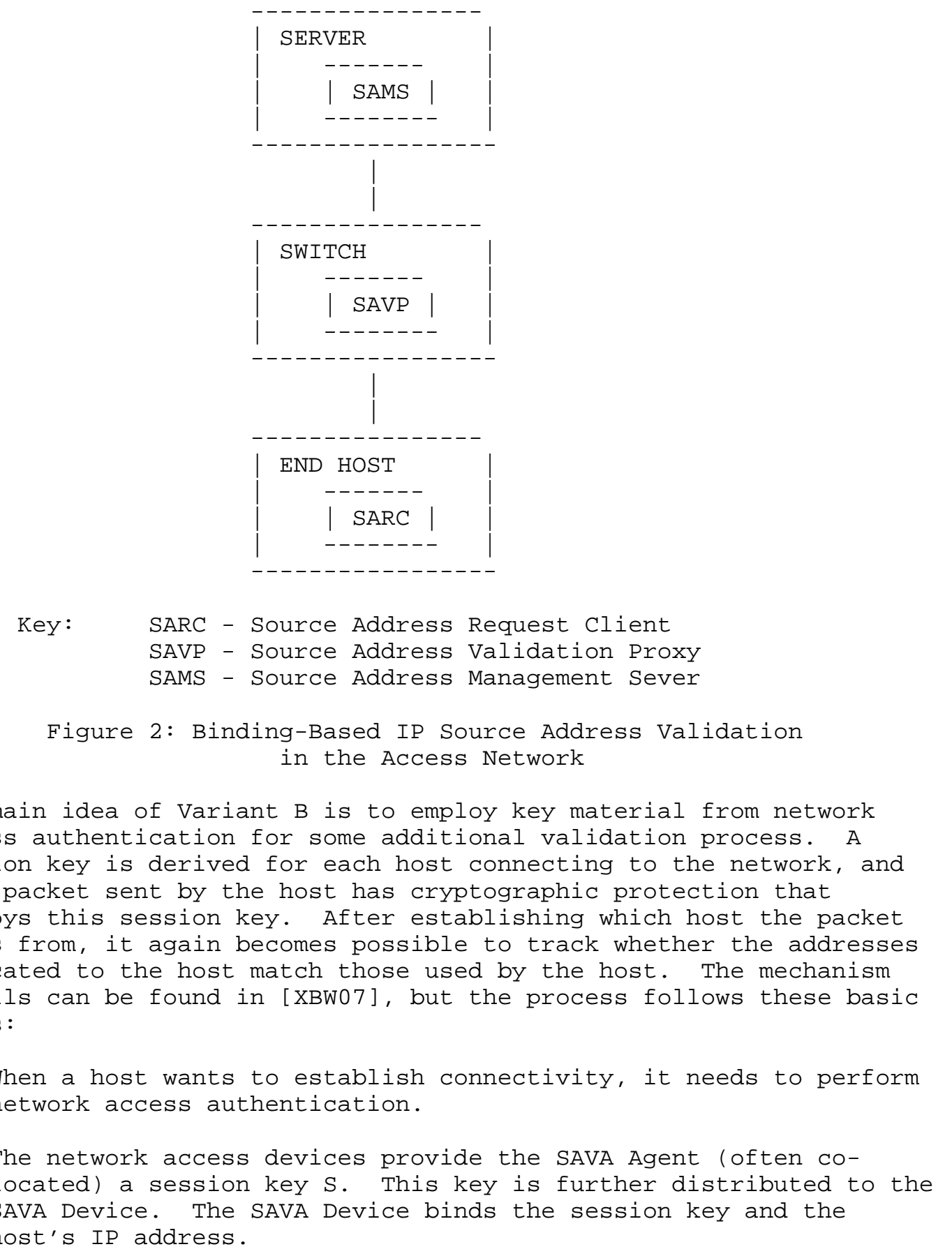


3. When the host sends packet $M$ to somewhere outside the access network, either the host or the SAVA Agent needs to generate a message authentication code for each using key $S$ and packet $M$.

In the prototype, the message authentication code is carried in an experimental IPv6 extension header.

4. The SAVA Device uses the session key to authenticate the signature carried in the packet so that it can validate the source address.

In our testbed, we implemented and tested both solutions. The switch-based solution has better performance, but the switches in the access network would need to be upgraded (usually the number of switches in an access network is large). The signature-based solution could be deployed between the host and the exit router, but it has some extra cost in inserting and validating the signature.

2.3. IP Source Address Validation at Intra-AS/Ingress Point

We adopted the solution of the source address validation of IP packets at ingress points described in [RFC2827] and [RFC3704]; the latter describes source address validation at the ingress points of multi-homed access networks.

\subsection{IP Source Address Validation in the Inter-AS Case (Neighboring AS)}

Our design for the Inter-AS Source Address Validation included the following characteristics: It should cooperate among different ASes with different administrative authorities and different interests. It should be lightweight enough to support high throughput and not to influence forwarding efficiency.

The inter-AS level of SAVA can be classified into two sub-cases:

- Two SAVA-compliant ASes exchanging traffic are directly connected;

- Two SAVA-compliant ASes are separated by one or more intervening, non-SAVA-compliant providers. 


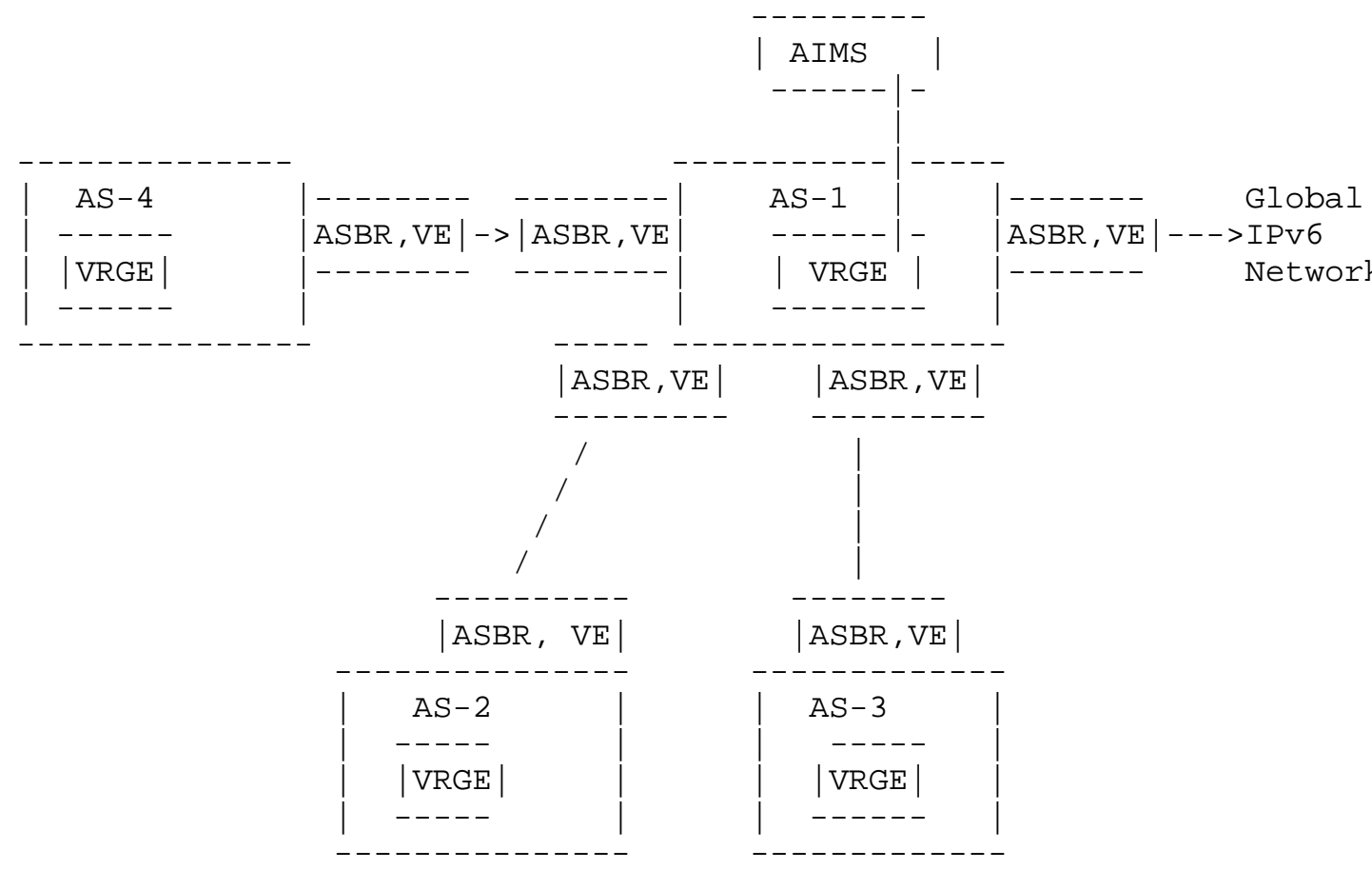

Key: $\quad$ AIMS - AS-IPv6 prefix Mapping Server

ASBR - AS Border Router

$\mathrm{VE}$ - Validating Engine

VR - Validation Rule

VRGE - Validation Rule Generating Engine

Figure 3: Inter-ISP (Neighboring AS) Solution

Two ASes that exchange traffic have a customer-to-provider, providerto-customer, peer-to-peer, or sibling-to-sibling relationship. In a customer-to-provider or provider-to-customer relationship, the customer typically belongs to a smaller administrative domain that pays a larger administrative domain for access to the rest of Internet. The provider is an AS that belongs to the larger administrative domain. In a peer-to-peer relationship, the two peers typically belong to administrative domains of comparable size and find it mutually advantageous to exchange traffic between their respective customers. Two ASes have a sibling-to-sibling relationship if they belong to the same administrative domain or to administrative domains that have a mutual-transit agreement. 
An AS-relation-based mechanism is used for neighboring SAVA-compliant ASes. The basic ideas of this AS-relation-based mechanism are as follows. It builds a VR table that associates each incoming interface of a router with a set of valid source address blocks, and then uses it to filter spoofed packets.

In the solution implemented on the testbed, the solution for the validation of IPv6 prefixes is separated into three functional modules: The Validation Rule Generating Engine (VRGE), the Validation Engine (VE), and the AS-IPv6 prefix Mapping Server (AIMS). Validation rules that are generated by the VRGE are expressed as IPV6 address prefixes.

The VRGE generates validation rules that are derived according to Table 1, and each AS has a VRGE. The VE loads validation rules generated by VRGE to filter packets passed between ASes (in the case of Figure 3, from neighboring ASes into AS-1). In the SAVA testbed, the VE is implemented as a simulated layer-2 device on a Linux-based machine inserted into the data path just outside each ASBR interface that faces a neighboring AS. In a real-world implementation, it would probably be implemented as a packet-filtering set on the ASBR. The AS-IPv6 prefix mapping server is also implemented on a Linux machine and derives a mapping between an IPv6 prefix and the AS number of that prefix.

\begin{tabular}{|c|c|c|c|c|c|}
\hline To $\backslash$ Export & $\begin{array}{l}\text { Own } \\
\text { Address }\end{array}$ & $\begin{array}{l}\text { Customer's } \\
\text { Address }\end{array}$ & $\begin{array}{l}\text { Sibling's } \\
\text { Address }\end{array}$ & $\begin{array}{l}\text { Provider's } \\
\text { Address }\end{array}$ & $\begin{array}{l}\text { Peer's } \\
\text { Address }\end{array}$ \\
\hline Provider & Y & Y & Y & & \\
\hline Customer & Y & Y & Y & Y & Y \\
\hline Peer & Y & Y & Y & & \\
\hline Sibling & Y & $Y$ & $Y$ & $Y$ & $\mathrm{Y}$ \\
\hline
\end{tabular}

\section{Table 1: AS-Relation-Based Inter-AS Filtering}

Different ASes exchange and transmit VR information using the ASRelation-Based Export Rules in the VRGE. As per Table 1, an AS exports the address prefixes of itself, its customers, its providers, its siblings, and its peers to its customers and siblings as valid prefixes, while it only exports the address prefixes of itself, its customers, and its siblings to its providers and peers as valid prefixes. With the support of the AS-IPv6 prefix mapping server, only AS numbers of valid address prefixes are transferred between ASes, and the AS number is mapped to address prefixes at the VRGE. 
Only changes of AS relation and changes of IP address prefixes belonging to an AS require the generation of VR updates.

The procedure's principal steps are as follows (starting from AS-1 in Figure 3):

1. When the VRGE has initialized, it reads its neighboring SAVAcompliant AS table and establishes connections to all the VEs in its own AS.

2. The VRGE initiates a VR renewal. According to its export table, it sends its own originated VR to VRGEs of neighboring ASes. In this process, VRs are expressed as AS numbers.

3. When a VRGE receives a new VR from its neighbor, it uses its own export table to decide whether it should accept the VR and, if it accepts a VR, whether or not it should re-export the VR to other neighboring ASes.

4. If the VRGE accepts a VR, it uses the AIMS to transform the ASexpressed VR into an IPv6 prefix-expressed VR.

5. The VRGE pushes the VR to all the VEs in its AS.

The VEs use these prefix-based VRs to validate the source IP addresses of incoming packets.

2.5. IP Source Address Validation in the Inter-AS Case (Non-Neighboring AS)

In the case where two ASes do not exchange packets directly, it is not possible to deploy a solution like that described in the previous section. However, it is highly desirable for non-neighboring ISPS to be able to form a trust alliance such that packets leaving one AS will be recognized by the other and inherit the validation status they possessed on leaving the first AS. There is more than one way to do this. For the SAVA experiments to date, an authentication tag method has been used. This solution is inspired by the work of [Brem05].

The key elements of this lightweight authentication tag based mechanism are as follows: For each pair of SAVA-compliant ASes, there is a pair of unique temporary authentication tags. All SAVAcompliant ASes together form a SAVA AS Alliance. When a packet is leaving its own AS, if the destination IP address belongs to an AS in the SAVA AS Alliance, the edge router of this AS looks up the authentication tag using the destination AS number as the key, and adds an authentication tag to the packet. When a packet arrives at 
the destination AS, if the source address of the packet belongs to an AS in the SAVA AS Alliance, the edge router of the destination AS searches its table for the authentication tag using the source AS number as the key, and the authentication tag carried in the packet is verified and removed. As suggested by its name, this particular method uses a lightweight authentication tag. For every packet forwarded, the authentication tag can be put in an IPv6 hop-by-hop extension header. It is reasonable to use a 128-bit shared random number as the authentication tag to save the processing overhead brought by using a cryptographic method to generate the authentication tag.

The benefit of this scheme compared to merely turning on local address validation (such as RFC 2827) is as follows: when local address validation is employed within a group of networks, it is assured that their networks do not send spoofed packets. But other networks may still do this. With the above scheme, however, this capability is eliminated. If someone outside the alliance spoofs a packet using a source address from someone within the alliance, the members of the alliance refuse to accept such a packet.

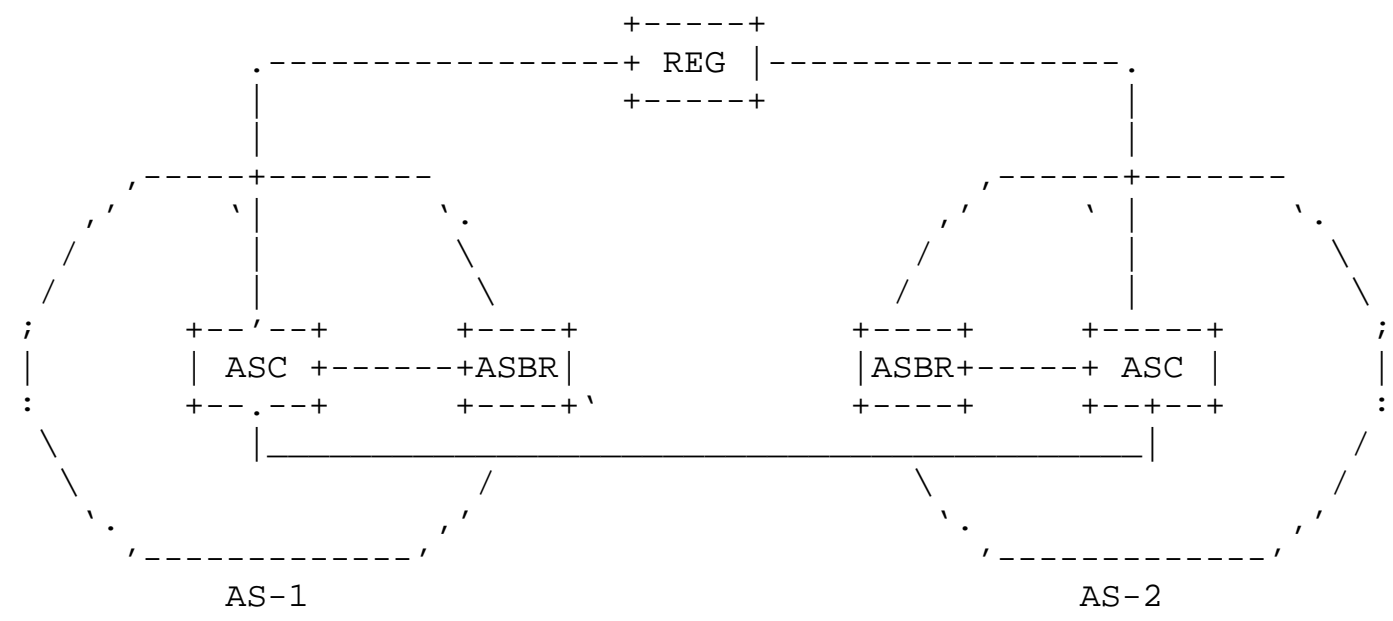

Key: $\quad \begin{aligned} & \text { REG - Registration Server } \\ & \text { ASC - AS Control Server } \\ & \text { ASBR - AS Border Router }\end{aligned}$

Figure 4: Inter-AS (Non-Neighboring AS) Solution

There are three major components in the system: the Registration Server (REG), the AS Control Server (ASC), and the AS Border Router $(\mathrm{ASBR})$. 
The Registration Server is the "center" of the trust alliance (TA). It maintains a member list for the TA. It performs two major functions:

- Processes requests from the AS Control Server, to get the member list for the TA.

- Notifies each AS Control server when the member list is changed.

Each AS deploying the method has an AS Control Server. The AS

Control server has three major functions:

- Communicates with the Registration Server, to get the up-to-date member list of $\mathrm{TA}$.

- Communicates with the AS Control Server in other member ASes in the TA, to exchange updates of prefix ownership information and to exchange authentication tags.

- Communicates with all AS Border Routers of the local AS, to configure the processing component on the AS Border Routers.

The AS Border Router does the work of adding the authentication tag to the packet at the sending AS, and the work of verifying and removing the authentication tag at the destination AS.

In the design of this system, in order to decrease the burden on the REG, most of the control traffic happens between ASCs.

The authentication tag needs to be changed periodically. Although the overhead of maintaining and exchanging authentication tags between AS pairs is $O(N)$ from the point of view of one AS, rather than $O\left(N^{\wedge} 2\right)$, the traffic and processing overhead do increase as the number of ASes increases. Therefore, an automatic authentication tag refresh mechanism is utilized in this solution. In this mechanism, each peer runs the same algorithm to automatically generate an authentication tag sequence. Then the authentication tag in packets can be changed automatically with high frequency. To enhance the security, a seed is used for the algorithm to generate an authentication tag sequence robust against guessing. Thus, the peers need only to negotiate and change the seed at very low frequency. This lowers the overhead associated with frequently negotiating and changing the authentication tag while maintaining acceptable security.

Since the authentication tag is put in an IPv6 hop-by-hop extension header, the MTU issues should be considered. Currently we have two solutions to this problem. Neither of the solutions is perfect, but 
they are both feasible. One possible way is to set the MTU at the ASBR to be 1280 bytes, which is the minimum MTU for the IPv6. Thus, there should be no ICMP "Packet Too Big" message received from the downstream gateways. The disadvantage of this solution is that it doesn't make good use of the available MTU. The other possible way is to let the ASBR catch all incoming ICMP "Packet Too Big" messages, and decrease the value in the MTU field before forwarding it into the AS. The advantage of this solution is that it can make good use of the available MTU. But such processing of ICMP packets at the ASBR may create a target for a denial-of-service (DoS) attack.

Because the authentication tag is validated at the border router of the destination AS, not the destination host, the destination options header is not chosen to carry the authentication tag.

Authentication tag management is a critical issue. Our work focused on two points: tag negotiation and tag refresh. The tag negotiation happens between the ASCs of a pair of ASes in the SAVA AS Alliance. Considering the issue of synchronization and the incentive of enabling SAVA, receiver-driven tag negotiation is suggested. It gives more decision power to the receiver AS rather than the sender AS. With a receiver-driven scheme, the receiver AS can decide the policies of tag management. The packets tagged with old authentication tags should not be allowed indefinitely. Rather, after having negotiated a new tag, the old tag should be set to be invalid after a period of time. The length of this period is a parameter that will control how long the old tag will be valid after the new tag has been assigned. In the experiment, we used five seconds.

The trust alliance is intended to be established dynamically (join and quit), but in this testbed we needed to confirm off-line the initial trust among alliance members.

\section{SAVA Testbed}

\subsection{CNGI-CERNET2}

The prototypes of our solutions for SAVA are implemented and tested on CNGI-CERNET2. CNGI-CERNET2 is one of the China Next Generation Internet (CNGI) backbones, operated by the China Education and Research Network (CERNET). CNGI-CERNET2 connects 25 core nodes distributed in 20 cities in China at speeds of $2.5-10 \mathrm{~Gb} / \mathrm{s}$. The CNGI-CERNET2 backbones are IPV6-only networks rather than being a mixed IPv4/IPv6 infrastructure. Only some Customer Premises Networks (CPNs) are dual-stacked. The CNGI-CERNET2 backbones, CNGI-CERNET2 CPNs, and CNGI-6IX all have globally unique AS numbers. Thus a multi-AS testbed environment is provided. 


\subsection{SAVA Testbed on CNGI-CERNET2 Infrastructure}

It is intended that eventually the SAVA testbed will be implemented directly on the CNGI-CERNET2 backbone, but in the early stages the testbed has been implemented across 12 universities connected to CNGI-CERNET2. First, this is because some of the algorithms need to be implemented in the testbed routers themselves, and to date they have not been implemented on any of the commercial routers forming the CNGI-CERNET2 backbone. Second, since CNGI-CERNET2 is an operational backbone, any new protocols and networking techniques need to be tested in a non-disruptive way.

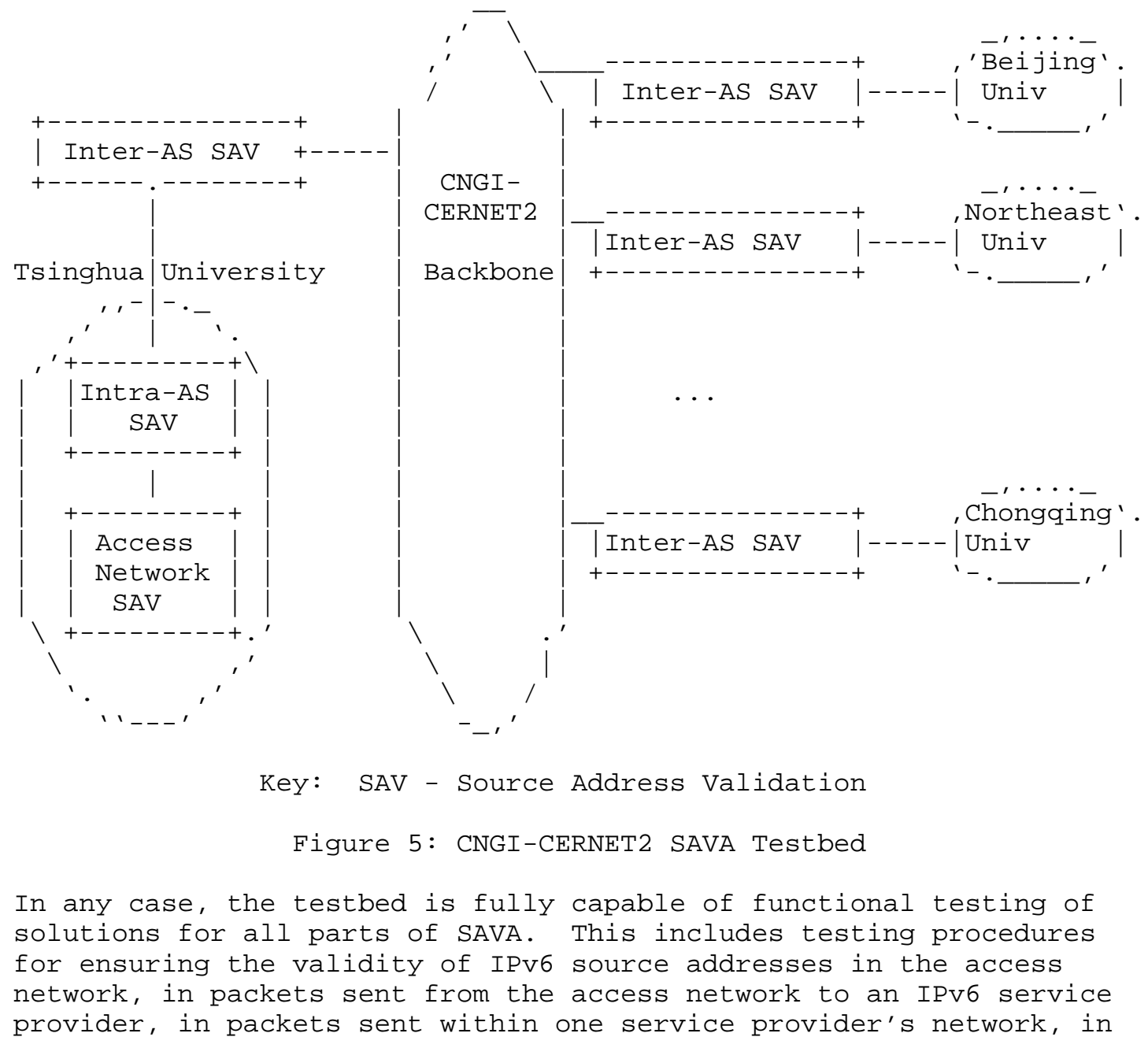

Wu, et al.

Experimental

[Page 16] 
packets sent between neighboring service providers, and in packets sent between service providers separated by an intervening transit network.

The testbed is distributed across 12 universities connected to CNGICERNET2.

Each of the university installations is connected to the CNGI-CERNET2 backbone through a set of inter-AS Source Address Validation prototype equipment and traffic monitoring equipment for test result display.

Each university deployed one AS. Six universities deployed all parts of the solution and are hence fully-featured, with validation at the inter-AS, intra-AS, and access network levels all able to be tested. In addition, a suite of applications that could be subject to spoofing attacks or that can be subverted to carry out spoofing attacks were installed on a variety of servers. Two solutions for the access network were deployed.

\section{Test Experience and Results}

The solutions outlined in section 2 were implemented on the testbed described in section 3. Successful testing of all solutions was been carried out, as detailed in the following sections.

\subsection{Test Scenarios}

For each of the test scenarios, we tested many cases. Taking the Inter-AS (non-neighboring AS) SAVA solution test as an example, we classified the test cases into three classes: normal class, dynamic class, and anti-spoofing class.

1. For normal class, there are three cases: Adding authentication tag Test, Removing authentication tag Test, and Forwarding packets with valid source address.

2. For dynamic class, there are four cases: Updating the authentication tag between ASes, The protection for a newly joined member AS, Adding address space, and Deleting address space.

3. For anti-spoofing class, there is one case: Filtering of packets with forged IP addresses.

As is shown in Figure 5, we have "multiple-fence" design for our SAVA testbed. If source address validation is deployed in the access network, we can get a host granularity validation. If source address 
validation is deployed at the intra-AS level, we can guarantee that the packets sent from this point have a correct IP prefix. If source address validation is deployed at the inter-AS level, we can guarantee that the packets sent from this point are from the correct AS.

\subsection{Test Results}

1. The test results are consistent with the expected ones. For an AS that has fully-featured SAVA deployment with validation at the inter-AS, intra-AS, and access network levels, packets that do not hold an authenticated source address will not be forwarded in the network. As a result, it is not possible to launch network attacks with spoofed source addresses. Moreover, the traffic in the network can be traced back accurately.

2. For the Inter-AS (non-neighboring AS) SAVA solution, during the period of authentication tag update, the old and the new authentication tags are both valid for source address validation; thus, there is no packet loss.

3. For the Inter-AS (non-neighboring AS) SAVA solution, the validation function is implemented in software on a device running Linux, which simulates the source address validation functions of a router interface. It is a layer-2 device because it needs to be transparent to the router interface. During the test, when the devices were connected directly, normal line-rate forwarding was achieved. When the devices were connected with routers from another vendor, only a very limited forwarding speed was achieved. The reason is that the authentication tags are added on the IPv6 hop-by-hop option header, and many current routers can handle the hop-by-hop options only at a limited rate.

5. Limitations and Issues

There are several issues both within this overall problem area and with the particular approach taken in the experiment.

\subsection{General Issues}

There is a long-standing debate about whether the lack of universal deployment of source address validation is a technical issue that needs a technical solution, or if mere further deployment of existing tools (such as RFC 2827) would be a more cost effective way to improve the situation. Further deployment efforts of this tool have proved to be slow, however. Some of the solutions prototyped in this experiment allow a group of network operators to have additional protection for their networks while waiting for universal deployment 
of simpler tools in the rest of the Internet. This allows them to prevent spoofing attacks that the simple tools alone would not be able to prevent, even if already deployed within the group.

Similarly, since a large fraction of current denial-of-service attacks can be launched by employing legitimate IP addresses belonging to botnet clients, even universal deployment of better source address validation techniques would be unable to prevent these attacks. However, tracing these attacks would be easier given that there would be more reliance on the validity of source address.

There is also a question about the optimal placement of the source address validation checks. The simplest model is placing the checks on the border of a network. Such RFC 2827-style checks are more widely deployed than full checks ensuring that all addresses within the network are correct. It can be argued that it is sufficient to provide such coarse granularity checks, because this makes it at least possible to find the responsible network administrators. However, depending on the type of network in question, those administrators may or may not find it easy to track the specific offending machines or users. It is obviously required that the administrators have a way to trace offending equipment or users -even if the network does not block spoofed packets in real-time.

New technology for address validation would also face a number of deployment barriers. For instance, all current technology can be locally and independently applied. A system that requires global operation (such as the Inter-AS validation mechanism) would require significant coordination, deployment synchronization, configuration, key setup, and other issues, given the number of ASes.

Similarly, deploying host-based access network address validation mechanisms requires host changes, and can generally be done only when the network owners are in control of all hosts. Even then, the changing availability of the host for all types of products and platforms would likely prevent universal deployment even within a single network.

There may be also be significant costs involved in some of these solutions. For instance, in an environment where access network authentication is normally not required, employing an authenticationbased access network address validation would require deployment of equipment capable of this authentication as well as credentials distribution for all devices. Such undertaking is typically only initiated after careful evaluation of the costs and benefits involved. 
Finally, all the presented solutions have issues in situations that go beyond a simple model of a host connecting to a network via the same single interface at all times. Multihoming, failover, and some forms of mobility or wireless solutions may collide with the requirements of source address validation. In general, dynamic changes to the attachment of hosts and topology of the routing infrastructure are something that would have to be handled in a production environment.

\subsection{Security Issues}

The security vs. scalability of the authentication tags in the Inter-AS (non-neighboring AS) SAVA solution presents a difficult tradeoff. Some analysis about the difficulty of guessing the authentication tag between two AS members was discussed in [Brem05]. It is relatively difficult, even with using a random number as an "authentication tag". The difficulty of guessing can be increased by increasing the length of the authentication tag.

In any case, the random number approach is definitely vulnerable to attackers who are on the path between the two ASes.

On the other hand, using an actual cryptographic hash in the packets will cause a significant increase in the amount of effort needed to forward a packet. In general, addition of the option and the calculation of the authentication tag consume valuable resources on the forwarding path. This resource usage comes on top of everything else that modern routers need to do at ever increasing line speeds. It is far from clear that the costs are worth the benefits.

\subsection{Protocol Details}

In the current CNGI-CERNET2 SAVA testbed, a 128-bit authentication tag is placed in an IPv6 hop-by-hop option header. The size of the packets increases with the authentication tags. This by itself is expected to be acceptable, if the network administrator feels that the additional protection is needed. The size increases may result in an MTU issue, and we found a way to resolve it in the testbed. Since an IPv6 hop-by-hop option header was chosen, the option header has to be examined by all intervening routers. While in theory this should pose no concern, the test results show that many current routers handle hop-by-hop options with a much reduced throughput compared to normal traffic.

The Inter-AS (neighboring AS) SAVA solution is based on the AS relation; thus, it may not synchronize with the dynamics of route changes very quickly and it may cause false positives. Currently, 
CNGI-CERNET2 is a relatively stable network, and this method works well in the testbed. We will further study the impact of false positives in an unstable network.

The access network address validation solution is merely one option among many. Solutions appear to depend highly on the chosen link technology and network architecture. For instance, source address validation on point-to-point links is easy and has generally been supported by implementations for years. Validation in shared networks has been more problematic, but is increasing in importance given the use of Ethernet technology across administrative boundaries (such as in DSL). In any case, the prototyped solution has a number of limitations, including the decision to use a new address configuration protocol. In a production environment, a solution that is suitable for all IPv6 address assignment mechanisms would be needed.

\section{Conclusion}

Several conclusions can be drawn from the experiment.

First, the experiment is a proof that a prototype can be built that is deployable on loosely-coupled domains of test networks in a limited scale and "multiple-fence" design for source address validation. The solution allows different validation granularities, and also allows different providers to use different solutions. The coupling of components at different levels of granularity can be loose enough to allow component substitution.

Incremental deployment is another design principle that was used in the experiment. The tests have demonstrated that benefit is derived even when deployment is incomplete, thus giving providers an incentive to be early adopters.

The experiment also provided a proof of concept for the switch-based local subnet validation, network authentication based validation, filter-based Inter-AS validation, and authentication tag-based Inter-AS validation mechanisms. The client host and network equipment need to be modified and some new servers should be deployed.

Nevertheless, as discussed in the previous section, there are a number of limitations, issues, and questions in the prototype designs and the overall source address validation space. 
It is our hope that some of the experiences will help vendors and the Internet standards community in these efforts. Future work in this space should attempt to answer some of the issues raised in Section 5. Some of the key issues going forward include:

- Scalability questions and per-packet operations.

- Protocol design issues, such as integration to existing address allocation mechanisms, use of hop-by-hop headers, etc.

o Cost vs. benefit questions. These may be ultimately answered only by actually employing some of these technologies in production networks.

o Trust establishment issue and study of false positives.

- Deployability considerations, e.g. modifiability of switches, hosts, etc.

\section{Security Considerations}

The purpose of the document is to report experimental results. Some security considerations of the solution mechanisms of the testbed are mentioned in the document, but are not the main problem to be described in this document.

8. Acknowledgements

This experiment was conducted among 12 universities -- namely, Tsinghua University, Beijing University, Beijing University of Post and Telecommunications, Shanghai Jiaotong University, Huazhong University of Science and Technology in Wuhan, Southeast University in Nanjing, South China University of Technology in Guangzhou, Northeast University in Shenyang, Xi'an Jiaotong University, Shandong University in Jinan, University of Electronic Science and Technology of China in Chengdu, and Chongqing University. The authors would like to thank everyone involved in this effort in these universities.

The authors would like to thank Jari Arkko, Lixia Zhang, and Pekka Savola for their detailed review comments on this document, and thank Paul Ferguson and Ron Bonica for their valuable advice on the solution development and the testbed implementation. 
9. References

9.1. Normative References

[RFC2827] Ferguson, P. and D. Senie, "Network Ingress Filtering: Defeating Denial of Service Attacks which employ IP Source Address Spoofing", BCP 38, RFC 2827, May 2000.

[RFC3704] Baker, F. and P. Savola, "Ingress Filtering for Multihomed Networks", BCP 84, RFC 3704, March 2004.

\subsection{Informative References}

[Brem05] Bremler-Barr, A. and H. Levy, "Spoofing Prevention Method", INFOCOM 2005.

[Li02] Li, J., Mirkovic, J., Wang, M., Reiher, P., and L. Zhang, "SAVE: Source Address Validity Enforcement Protocol", INFOCOM 2002 .

[Park01] Park, K. and H. Lee, "On the effectiveness of route-based packet filtering for distributed Dos attack prevention in power-law internets", SIGCOMM 2001.

[Snoe01] Snoeren, A., Partridge, C., Sanchez, L., and C. Jones, "A Hash-based IP traceback", SIGCOMM 2001.

[Wu07] Wu, J., Ren, G., and X. Li, "Source Address Validation: Architecture and Protocol Design", ICNP 2007.

[XBW07] Xie, L., Bi, J., and J. Wu, "An Authentication based Source Address Spoofing Prevention Method Deployed in IPv6 Edge Network", ICCS 2007. 


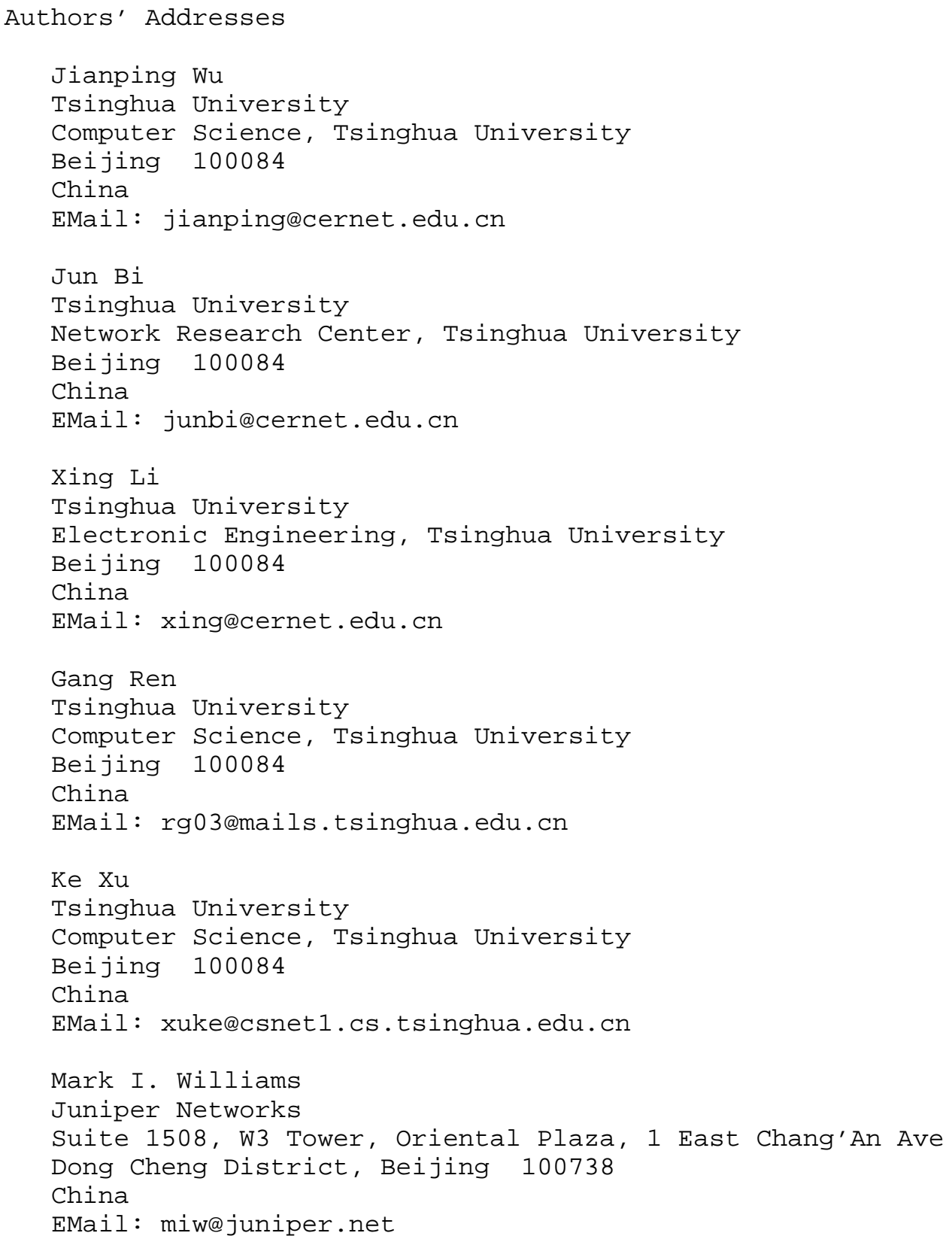


Full Copyright statement

Copyright (C) The IETF Trust (2008).

This document is subject to the rights, licenses and restrictions contained in BCP 78, and except as set forth therein, the authors retain all their rights.

This document and the information contained herein are provided on an "AS IS" basis and THE CONTRIBUTOR, THE ORGANIZATION HE/SHE REPRESENTS OR IS SPONSORED BY (IF ANY), THE INTERNET SOCIETY, THE IETF TRUST AND THE INTERNET ENGINEERING TASK FORCE DISCLAIM ALL WARRANTIES, EXPRESS OR IMPLIED, INCLUDING BUT NOT LIMITED TO ANY WARRANTY THAT THE USE OF THE INFORMATION HEREIN WILL NOT INFRINGE ANY RIGHTS OR ANY IMPLIED WARRANTIES OF MERCHANTABILITY OR FITNESS FOR A PARTICULAR PURPOSE.

Intellectual Property

The IETF takes no position regarding the validity or scope of any Intellectual Property Rights or other rights that might be claimed to pertain to the implementation or use of the technology described in this document or the extent to which any license under such rights might or might not be available; nor does it represent that it has made any independent effort to identify any such rights. Information on the procedures with respect to rights in RFC documents can be found in BCP 78 and BCP 79 .

Copies of IPR disclosures made to the IETF Secretariat and any assurances of licenses to be made available, or the result of an attempt made to obtain a general license or permission for the use of such proprietary rights by implementers or users of this specification can be obtained from the IETF on-line IPR repository at http://www.ietf.org/ipr.

The IETF invites any interested party to bring to its attention any copyrights, patents or patent applications, or other proprietary rights that may cover technology that may be required to implement this standard. Please address the information to the IETF at ietf-ipreietf.org. 